\title{
Comments on the current treatment of people with grave's hyperthyroidism
}

\section{Opinión}

Graves' hyperthyroidism (GH) is the most common cause of hyperthyroidism in iodine-sufficient areas. The cause of GH is an unregulated stimulation of the thyroid-stimulating-hormone (TSH) receptor by autoreactive TSH-receptor antibodies (TRAbs). Patients with uncontrolled hyperthyroidism had an increased risk of mortality and substantial cardiovascular morbidity, including arrhythmias, stroke and heart failure. ${ }^{1}$

I want to clarify that as a clinician I am going to talk about some aspects of the treatment of patients with Graves hyperthyroidism, but not about the treatment of Graves' disease, which is something different. The treatment of these people is not satisfactory, because the target of the different treatments does not affect the etiopathogenic bases of the disease, and the consequence is that a high percentage of patients have to cause a disease (hypothyroidism) to treat the first (hyperthyroidism).

Thionamide derived drugs (ATD), radioiodine (RAI) and total thyroidectomy (TT) are the main treatment options. All of them proven to be effective, but they also have important drawbacks, so it is a challenge for both doctors and patients to choose one of these treatment modalities, for this reason the main guidelines for management of GH patients recommend an active discussion between the patients and doctors regarding the benefits, risks and logistics of the different treatment modalities, ${ }^{2,3}$ always taken into consideration the patient' preference.

Currently, antithyroid drugs Methimazole (MMI) or Propylthiouracil (PTU) are the first treatment option for these patients worldwide, ${ }^{4}$ mainly because compared to the other treatment options it preserves the thyroid gland, since is well-known that one of the characteristics of autoimmune diseases, is to have spontaneously periods of increase and others of remission of the disease. Other reason for the pharmacological treatment is of first choice is the fact that in the last 3 decades it is observed that the phenotype of patients with GH is milder than in the past ${ }^{5}$ and it is well known that patients with mild/ moderate forms of GH respond very well to treatment with ATD, However, the main reason for treat with ATD should be patient preference.

MMI is the first choice among antithyroid drugs, except in patients during the first trimester of pregnancy. Initial doses of $20-30 \mathrm{mg} /$ day and maintenance doses of 5-10mg usually enough in most cases. The minimum duration must be at least 12 to 18 months; it is advisable to continue the treatment until the antibody values against the TSH receptors (TRAbs) have become negative.

The frequency of side effects of ATDs is around 5\%, mainly urticariform lesions, itching and less frequently liver lesions, mainly when propyl thiouracil is used; in general the side effects are dose dependent. ${ }^{6}$ The main problem with antithyroid treatment is the high rate of recurrence of the disease after discontinuation of treatment, which leads to $50 \%$. For this reason it has been tried to find some
Volume 8 Issue 2 - 2020

\author{
Ricardo V Garcia-Mayor \\ Endocrinology \& Nutrition Unit. HM Hospital of Vigo, Spain
}

Correspondence: Ricardo V Garcia-Mayor, Endocrinology \& Nutrition Unit. HM Hospital of Vigo, Manuel Olivie Street, 36203 Vigo, Spain, Email rvgarciamayo@hmhospitales.com, Ricardo.V.Garcia-Mayor.Garci@sergas.es

Received: February 23, 2020 | Published: March 06, 2020

clinical, biochemical or genetic markers that determined before starting treatment could predict that patients would have long-term disease remissions, after the suppression of the medication. ${ }^{7-9}$ Mild hyperthyroidism, absence of orbitopathy, minor thyroid volume, no smoking habit or same genetic markers are associate with high remission rate. Unfortunately these parameters have little value when we are in front of a specific patient.

The main indications for dealing with RAI are: patient preference, toxic effects to ATD, and patients with other comorbidities such as cardiovascular, respiratory, liver diseases, etc. The main limitation of RAI treatment are the presence of orbitopathy and that results in athyreotic hypothyroidism that is no ease to management. Recent studies found that RAI for hyperthyroidism is associated with increased solid cancer mortality, ${ }^{10}$ and that patients treated with RAI compared to ATD and Thyroidectomy have worse quality of life at 6-10years after of RAI treatment. ${ }^{11}$

Finally, total thyroidectomy (TT) is indicated for patients with big or/and compressive goitre and GH associated with malignant nodules. The main problems of TT are: high dependence of surgeon qualification, athyreotic hipothyroidism and high initial cost.

\section{Conclusion}

In conclusion, unless the patients have the opposite opinion, the first option to treat Graves hyperthyroidism is with antithyroid drugs, which can be equally useful when the disease relapse. ${ }^{12}$

\section{Acknowledgments}

None.

\section{Conflicts of interest}

The authors declare that there is no conflict of interest. 


\section{Funding}

None.

\section{References}

1. Brandt F, Green A, Hegedüs L, et al. A critical review and meta-analysis of the association between overt hyperthyroidism and mortality. Eur $J$ Endocrinol. 2011;165(4):491-497.

2. Ross DS, Burch HB, Cooper DS, et al. American Association Guidelines for diagnosis and management of hypertyroidism and other causes of thyrotoxicosis. Thyroid. 2016;26(10):1343-1421.

3. Kahaly GJ, Bartalena L, Hegedüs L, et al. European Thyroid Association guideline for the management of Graves' hyperthyroidism. Eur Thyroid J. 2018;7(4):167-186.

4. Brito JP, Schilz S, Singh Ospina N, et al. Antithyroid drugs the most common treatment for Graves' disease in the United States: A nationwide population-based study. Thyroid. 26;26(8):1144-1145.

5. Bartalena L, Masiello E, Magri F, et al. The phenotype of newly diagnosed Graves' disease in Italy in recent years is milder than in the past: results of a large observational longitudinal study. J Endocrinol Invest. 2016;39(12):1445-1451.

6. Garcia-Mayor RV, Larranaga A. Treatment of Graves' hyperthyroidism with thionamide-derived drugs: Review. Medicinal Chemistry. 2010;6(4):239-246.
7. Garcia-Mayor RV, Paramo C, Luna Cano R, et al. Antithyroid drug and Graves' hyperthyroidism: Significance of treatment duration and TRAb determination on lasting remission. $J$ Endocirnol Invest. 1992;15(11):815-820.

8. Garcia-Mayor RV, Alvarez Vazquez P, Fluiters E, et al. Long-term remission after antithyroid drug withdrawal in patients with Graves' hyperthyroidism: parameters with prognostic value. Endocrine. 2019;63(2):316-322.

9. Masiello E, Veronesi G, Gallo D, et al. Antithyroid drug treatment for Graves' hyperthyroidism: baseline predictive models of relapse after treatment for a patient-tailored management. J Endocrinol Invest. 2018;41(12):1425-1432.

10. Kitahara C, Berrington de González A, Bouville A, et al. Association of radioactive iodine treatment with cancer mortality in patients with hyperthyroidismo. JAMA Intern Med. 2019;179(8):1034-1042.

11. Töring O, Watt Tm Sjölin G, Byström K, et al. Impaired quality of life after radioiodine therapy compared with antithyroids drugs or surgical treatment for Graves' hyperthyroidism: a long-term follow-up with the Thyroid-Related Patient-Reported Outcome Questionnaire and 36Item Short Form Heath Status Survey. Thyroid. 2019;29(3):322-331.

12. Garcia-Mayor RV. Reasons to consider low-dose Metimazole as the best option to treat patients with recurrent Graves' hypethyroidism. Rev Clin Esp. 2019;219(3):168-170. 